\title{
La parodia nei romanzi di Elsa Morante
}

\author{
Lucia DELL'AiA ${ }^{1}$ \\ Università degli Studi di Bari "Aldo Moro" \\ lucia.dellaia@virgilio.it \\ 1.dellaia@lettere.uniba.it
}

\begin{abstract}
RIASSUNTO
Si prende in esame la complessiva produzione romanzesca di Elsa Morante, da Menzogna e sortilegio ad Aracoeli, cercando di trovare una cifra interpretativa complessiva della sua scrittura grazie al concetto di parodia, anche attraverso una ricognizione che coinvolge $\mathrm{i}$ termini chiave della poetica della scrittrice: mistero, ambiguità, scandalo. Per indagare la nozione di parodia, la mediazione filosofica più significativa che questa ricerca ha utilizzato è la teoria del romanzo di Gianni Carchia, utile al fine di dimostrare l'intimo legame che nei romanzi di Morante si stabilisce fra mito e storia.
\end{abstract}

Parole chiave: Parodia, scandalo, mito, storia, Gianni Carchia.

\section{The Parody in Elsa Morante's Novels}

\begin{abstract}
This paper analyses the whole fictional work of Elsa Morante, from Menzogna e Sortilegio to Aracoeli, using the concept of parody as a parameter of interpretation, also investigating the keywords of Morante's poetics (e.g. mistery, ambiguity, scandal). In conducting this analysis, the main theoretical and philosophical framework has been taken from Gianni Carchia's theory of the novel which has been used to demonstrate the strong and deep connection between myth and history in Morante's novels.
\end{abstract}

Key words: Parody, Scandal, Myth, History, Gianni Carchia.

La tesi contenuta in questa relazione è il frutto di uno studio, che procede ormai da alcuni anni, intorno alla specificità della nozione di narrazione nella scrittura di Elsa Morante ${ }^{2}$. Dopo varie indagini critiche intorno all'argomento, da me condotte avvalendomi di varie interpretazioni della forma romanzo, di recente mi è sembrato di trovare in una specifica teoria del romanzo, elaborata dal filosofo Gianni Carchia, e poco nota nell'ambito degli studi teorici sull'argomento, un interessante modello per comprendere l'originalità della scrittura romanzesca della scrittrice e, allo stesso

\footnotetext{
${ }^{1}$ Università degli Studi di Bari "Aldo Moro".

${ }^{2}$ Rimando soprattutto alla mia monografia sul Mondo salvato dai ragazzini (Dell'Aia 2013) e al saggio La Storia e l'affabulazione (Dell'Aia 2012).
} 
tempo, per individuare una possibile tradizione entro cui essa può essere inserita. Il punto centrale dell'elaborazione teorica di Carchia sta nel fatto di intendere il romanzo come genere, sin dalle sue origini, profondamente legato alla parodia, al mistero e alla dimensione del demonico: ciò mi ha consentito di spiegare, come vedremo, la complessa trama che nella scrittura di Morante si crea fra alcuni termini ricorrenti, quali "parodia", "mistero", "ambiguità", "scandalo". La ricerca, dunque, è partita da una ricognizione prima di tutto terminologica, fatta sui quattro romanzi, per ricavare da quest'ultima una interpretazione generale della forma di scrittura morantiana che pone al centro la parodia nel suo legame con la dimensione mitica e storica.

Che il termine "parodia" sia usato nell'Isola di Arturo, oltre che come sottotitolo della Serata a Colono, con l'intenzione di fare riferimento ad una specifica forma letteraria è una acquisizione certa nell'ambito degli studi su Elsa Morante dopo le riflessioni svolte in merito da Concetta D'Angeli (D'Angeli 2003). Non privo di interesse, invece, sarebbe approfondire quale particolare nozione di parodia, in quanto cifra essenziale e costitutiva della scrittura dell'autrice, si può ricavare dall'intera sua produzione romanzesca. A supporto di una tale ipotesi non è certo sufficiente rilevare che il termine ricorre in tutti e quattro i suoi romanzi; tuttavia, tale occorrenza non può essere trascurata e può costituire, se non altro, il punto di partenza per prendere in esame una vera e propria costellazione terminologica, entro cui il termine "parodia" è inserito, che rimanda ad una serie di significati che cercheremo di far emergere dai romanzi della scrittrice.

È Giorgio Agamben ad aver messo in luce che nell'Isola di Arturo l'uso del termine «Parodia», scritto non a caso con la maiuscola, e usato come epiteto ingiurioso di un personaggio centrale del romanzo, Wilhelm Gerace, idolo e padre di Arturo, nasconde una «speciale intenzione allegorica» con la quale l'autrice «ha fatto di un genere letterario - la parodia - il protagonista del suo libro» (Agamben 2005: 39-40 $)^{3}$.

Il personaggio di Wilhelm Gerace è descritto nel romanzo con gli occhi incantati del figlio Arturo che lo considera un essere diverso da tutti gli altri uomini esistenti, consistendo la sua «supremazia» proprio nella «differenza, che era il suo più bel mistero» (Morante 2001a: 974). Una differenza di «statura» e di «colori», derivante dal fatto di essere nato da una madre tedesca, in cui Arturo vede «il segno d'una stirpe non terrestre: come s'egli fosse il fratello del sole e della luna» (Morante 2001a: 975). Il biondo e alto Wilhelm, con gli occhi d'un colore «turchinoviolaceo» (Morante 2001a: 975), è per Arturo depositario di ragioni sue proprie che egli non può interrogare: «Il suo broncio era maestoso come l'oscurarsi del giorno, indizio certo di eventi misteriosi, e importanti come la Storia Universale» (Morante 2001a: 975). E se anche qualcosa di umano, come la malattia, lo avesse mai attaccato, essa non sarebbe sembrata ad Arturo come «uno dei soliti accidenti della

${ }^{3}$ Sulla interpretazione della parodia in Agamben mi permetto di rimandare a Dell'Aia 2012b: 24-32. 
natura», ma piuttosto come un «mistero rituale, in cui Wilhelm Gerace era l'eroe» ed egli non avrebbe dubitato "che una qualche commozione del cosmo, dai paesaggi terrestri fino alle stelle, dovesse accompagnare questo mistero paterno» (Morante 2001a: 976). La «specie gloriosa e diversa» (Morante 2001a: 982) a cui appartiene il padre di Arturo avvolge il personaggio in un alone leggendario e mitico: se Wilhelm si divertiva a gridare delle frasi in tedesco in una piana fra alte rocce, producendo il fenomeno dell'eco, ad Arturo quelle parole sembravano «enigmi» o suoni provenienti da un duello epico (Morante 2001a: 976); se egli, nella sua «noncuranza imbronciata verso l'Eternità» (Morante 2001a: 978), «si allontanava nuotando lento lento, quasi abbracciato al mare, al mare come a una sposa» (Morante 2001a: 985), «il mare - per Arturo - è uno splendore indifferente, come Lui» (Morante 2001a: 986).

La noia che improvvisamente attanaglia suo padre spinge Arturo a mutare il suo atteggiamento di indifferenza nei confronti delle continue partenze e arrivi sull'isola di Wilhelm e a seguire «le tracce del suo mistero» (Morante 2001a: 1288) per comprenderne le ragioni. Ma entrare «fin dentro il teatro dei suoi misteri» (Morante 2001a: 1290) significa per Arturo apprendere che un giovane rinchiuso nel Penitenziario di Procida, usando «il linguaggio segreto dei segnali» (Morante 2001a: 1297), una specie di alfabeto Morse che Arturo credeva di condividere solo con suo padre, scaccia via Wilhelm Gerace dicendogli: «VATTENE, PARODIA» (Morante 2001a: 1299) ${ }^{4}$. Arturo comprende che si tratta di un «cupo e grande dileggio» (Morante 2001a: 1299) nei confronti di suo padre, ma non conosce il significato della parola e lo cerca su un dizionario trovando questa definizione: «Imitazione del verso altrui, nella quale ciò che in altri è serio si fa ridicolo, o comico, o grottesco» (Morante 2001a: 1299). Arturo comprende che quel padre per lui oggetto di venerazione riceveva le più vergognose umiliazioni e veniva ripudiato «come l'ultimo servo» (Morante 2001a: 1300); e anche quando il giovane amante del padre dice ad Arturo: «Tuo padre è una PARODIA» (Morante 2001a: 1324), a lui appare sempre «bello come un grande principe romanzesco». Arturo si ostina «a cercare in lui, assurdamente, con disperazione, quell'aspetto comico o grottesco che gli meritava [...] l'epiteto di parodia»; anzi, addirittura bramava «di riconoscere davvero in lui qualcosa di ridicolo», ma purtroppo nella sua persona non scorgeva che «grazia» (Morante 2001a: 1325). In conclusione del romanzo, Arturo è costretto ad ammettere che «la vita è rimasta un mistero» e che, se ripensa a Wilhelm Gerace, lo immagina invecchiato e imbruttito «che va e torna, solo, scombinato, adorando chi gli dice parodia» (Morante 2001a: 1366).

Il padre di Arturo è, da una parte, oggetto di adorazione quasi divina e, dall'altra, umiliato questuante d'amore. Il mistero e la perenne doppiezza e ambiguità di Wilhelm Gerace erano già stati profetizzati da Romeo l'Amalfitano, il suo benefattore. Interpretando un sogno, egli aveva affermato che «alle anime viventi possono toccare due sorti: c'è chi nasce ape, e chi nasce rosa». Mentre l'ape deve

\footnotetext{
${ }^{4}$ Maiuscolo nel testo originale.
} 
rubare alla rosa un po' di miele, la rosa ha già in se stessa il proprio miele e non deve cercare altrove «la cosa più dolce che innamora». Infatti, «la prima di tutte le rose è Dio». Tuttavia, talvolta, le rose «sospirano di solitudine» e «le rose ignoranti non capiscono i propri misteri». Secondo l'Amalfitano, l'ape è più fortunata della rosa, soprattutto l'Ape Regina. Wilhelm invece è nato «col destino più dolce e col destino più amaro», cioè di essere l'ape e la rosa insieme (Morante 2001a: 1017).

Dall'insistenza con cui il termine "mistero" è accostato da Arturo alla figura di suo padre, allegoria della Parodia, possiamo senz'altro ricavare che per comprendere la nozione di parodia in Elsa Morante dobbiamo interrogare il rapporto che essa intrattiene con il mistero. Anche in questo caso può essere molto utile sviluppare il suggerimento offerto da Agamben, secondo cui «del mistero non può che darsi parodia» (Agamben 2005: 44), dal momento che la parodia è la forma stessa assunta dal mistero nel momento in cui si tenta di rivelarlo (Agamben 2005: 45). Aver tentato di conoscere le ragioni del mistero paterno ha condotto Arturo a scoprire che suo padre è, allegoricamente, una parodia. Egli stesso ammette che «tutti quanti si occupavano di cose semplici, naturali», mentre invece egli, per parte sua, inseguiva dei «misteri terribili e straordinari, che forse nemmeno esistevano»e che, in fondo, non desiderava nemmeno sapere (Morante 2001a: 1293-1294). Egli è, inoltre, costretto a concludere che «spesso certi nostri affetti, che presumiamo magnifici, addirittura sovrumani, sono in realtà insipidi; solo un'amarezza terrestre, magari atroce, può, come il sale, suscitare il sapore misterioso della loro profonda mescolanza» (Morante 2001a: 1304).

A proposito dei rituali misterici dell'antichità, ad esempio quelli eleusini, Agamben scrive che in essi «l'uomo antico non apprendeva qualcosa - una dottrina segreta - su cui, in seguito, doveva tacere, ma faceva gioiosamente esperienza dello stesso ammutolire, $[\ldots]$ cioè della possibilità, aperta all'uomo, $[\ldots]$ di un'esistenza lietamente e intransitivamente in-fantile», cioè priva di parola, dal momento che l'iniziato alle visioni «riceveva un'impressione, ma non un insegnamento» (Agamben 2010: 13). Il fatto allora che Arturo, tentando di svelare il mistero paterno, abbia scoperto che si tratta di una parodia, non autorizza affatto a pensare ad essa nei termini della definizione che egli stesso ne dà nel romanzo, leggendola sul vocabolario. Non sembra, infatti, che essa abbia a che fare con il comico o con il grottesco o con il ridicolo, perché, come abbiamo già visto, Arturo non riceverà mai nessuna di queste impressioni da suo padre; e l'ambiguità, la doppiezza (il fatto di essere ape e rosa insieme), la mescolanza di caratteri umani e divini, che facevano di lui un mistero, rimarranno inalterate fino alla fine del romanzo.

Ad aver individuato il legame profondo che unisce la parodia al mistero è stato Gianni Carchia, il quale ha riflettuto sul fatto che «l'umanizzazione del divino» messa in atto dalla religiosità misterica presuppone «una forma della contaminazione, della mescolanza del divino e dell'umano, inconcepibile per la religiosità olimpica del mito». Perciò, il mistero è «una forma di parodia della religiosità mitica» (Carchia 1983a: 24-25). Risulta, inoltre, interessante ai fini del nostro discorso anche il fatto che Carchia individui nella forma romanzo che nasce in epoca tardo-antica il «luogo di fondazione del misterico», dal momento che la 
specificità di tale genere «sta tutta nel fatto che non è tanto il suo radicarsi nel suolo dei misteri ciò che può spiegarlo, ma al contrario esso stesso può spiegare il senso dei misteri, anzi fondarlo» (Carchia 1983a: 26). In altri termini, il romanzo fonda il senso dei misteri antichi perché ricostruisce nello spazio estetico un intero universo storico-filosofico nel quale l'elemento umano e terrestre costituisce un tramite della vicenda divina, se pure solo parodicamente. E tutto ciò è possibile, secondo Carchia, perché il romanzo nasce come «spazio cosciente della simulazione del mito» (Carchia 1983a: 26), essendo la sua specificità quella di essere il primo genere della letteratura occidentale a nascere in totale autonomia rispetto a qualsiasi fondamento mitico (Carchia 1983a: 15). Dopo il tramonto del mito, il romanzo tardo-antico è l'«apparenza gratuita distaccata da ogni fondamento nell'ethos e nel mito»; esso, perciò, riproduce «l'insignificanza della vita abbandonata dagli dèi olimpici». D'altra parte, però, questa apparenza è incapace «di porsi come compiuta in se stessa» e, rinnegando la sua autonomia, è «fin dall'inizio ridotta ad illusione», cioè alla dimensione dell'ineffettuale della vita (Carchia 1983a: 16), per contrastare la quale vi è «un ritorno dello spazio mitico nella forma distanziata della sua parodia» (Carchia 1983a: 23). Per tale ragione, il romanzo è espressione per eccellenza del «demonico», ovvero di quello spazio intermedio fra l'umano e il divino (Carchia 1983a: 17).

Ci sembra, allora, che la specificità della scrittura romanzesca della Morante possa essere meglio compresa se la si riconduce a questa interpretazione della tradizione del romanzo e ad un ordine di problemi che investe il rapporto fra parodia, mistero e mito. Ciò consente di inserire in una nuova trama di significati quella che all'inizio abbiamo definito una vera e propria costellazione terminologica ricorrente in tutti i romanzi della scrittrice: parodia, mistero, ambiguità. L'universo dei romanzi di Elsa Morante è popolato di mistero e di ambiguità, «senza la quale - come scrive in Menzogna e sortilegio - nulla piace» (Morante 2001b: 778). Esso appare affollato di figure demoniche, descritte come divinità mitiche trasfigurate parodicamente, dato che «le poche gioie di cui ci ha lasciati eredi il padre Adamo», scrive sempre in Menzogna e sortilegio, sono di «ibrida specie» (Morante 2001b: 290). La vita fuori dall'Eden, la vita non più popolata di divinità, e tuttavia anelante a questa integrità priva di mescolanza di piacere e di dolore, è l'oggetto costante della materia romanzesca di Elsa Morante.

Secondo la nostra interpretazione, dunque, nella terminologia morantiana l'ambiguità è anch'essa, come il mistero, strettamente connessa con la nozione di parodia. Se teniamo conto dell'interpretazione della parodia in Scaligero, il quale l'ha posta in relazione con la rapsodia, di cui costituisce il ribaltamento, essa è, secondo la sua etimologia, «ciò che sta a fianco del canto», ovvero è un canto che si aggiunge ad un altro canto. Nel nostro caso non ci interessa tanto insistere sui procedimenti formali di ribaltamento di un testo o di un genere particolare, quanto piuttosto indagare le ragioni per cui, come scrive Genette, il Faust di Mann si chiede: «Perché quasi tutte le cose mie si devono affacciare con la loro parodia? Perché mi deve sembrare che quasi tutti [...] i mezzi e le convenienze dell'arte possano oggidì servire soltanto alla parodia?» (Genette 1997: 366). È probabile, 
come scrive sempre Genette, che «l'umanità, che scopre in continuazione nuovi significati, non può sempre inventare nuove forme, ed è talvolta costretta ad attribuire un senso nuovo a forme antiche. Il numero di favole o di metafore di cui è capace l'immaginazione degli uomini è limitato, ma codeste contate invenzioni possono essere tutto per tutti» (Genette 1997: 470-471).

Che l'immaginazione creatrice della Morante attinga ad un variegato e multiforme universo mitico, favoloso, leggendario e romanzesco è constatazione verificabile in tutti i suoi romanzi ${ }^{5}$. Il fatto che tale tradizione di forme non possa emergere nei suoi romanzi se non in veste di parodia potremmo spiegarlo prendendo spunto da alcune considerazioni che Furio Jesi svolge intorno al rapporto fra evocazione mitica e parodia. Se, da una parte, lo spirito della parodia, «ironico e negatore», sembra non potersi conciliare con «l'ingenuità e la commozione che consentono l'affiorare delle immagini mitiche», d'altra parte, però, scrive sempre Jesi, «chi compie una parodia sembra, in fondo, non potersi staccare dall'oggetto parodiato», dal momento che, per un verso, vi è «una forza [che] lo induce a non riporvi più la sua fiducia ed a passare oltre», per altro verso ve ne è un'altra che «gli impedisce di ricacciare quell'oggetto nell'oblio e nel silenzio». La parodia, dunque, non consiste in un «netto superamento» o «abbandono»; e «nel vincolo che unisce il parodista all'oggetto della parodia è lecito riconoscere la sopravvivenza di un'antica commozione, le tracce di un amore contro cui si lotta, ma che non si può sopprimere» (Jesi 2002: 189). Anche Jesi, come Genette, riconosce nel Doktor Faustus di Thomas Mann la teorizzazione moderna della parodia. Da questo testo, infatti, ricava l'idea che è l'«amore» per forme del passato che non è più possibile conservare a determinare l'atteggiamento del parodista: «quell'amore, più o meno

\footnotetext{
${ }^{5}$ Vorrei qui solo accennare ad una possibile ipotesi interpretativa dell' Isola di Arturo, che mi propongo di sviluppare in altra sede, tenendo conto dell'universo favolistico e mitico a cui Furio Jesi fa riferimento in un suo articolo segnalatomi da Maurizio Monina, che desidero qui ringraziare per il generoso suggerimento. Parlando di «connessioni archetipiche» e facendo riferimento al mito-favola di Amore e Psiche, Jesi scrive: «Gli etnologi della scuola di Leningrado hanno giustamente notato che la condizione della sposa - Psiche nel mito, o la donna della fiaba - rispecchia quella delle fanciulle che, presso società cosiddette primitive, trascorrevano un certo periodo insieme con gli adolescenti sottoposti alla iniziazione. La casa in cui quegli adolescenti trascorrevano il periodo iniziatico era considerata un luogo dell'aldilà, del regno dei morti (gli iniziati ritualmente morivano e rinascevano): dunque, anche la permanenza della fanciulla laggiù era un soggiorno nell'Aldilà. Di qui, l'invisibilità dei servitori: nel regno dei morti, l'invisibilità è tipica. E di qui, soprattutto, l'invisibilità dello sposo, o il divieto di vedere lo sposo. Sia Psiche, sia le protagoniste delle favole, subiscono insomma la sorte della greca Kore, rapita nell'Aldilà dal dio infero Ades, il cui primo attributo è l'invisibilità. Abbandonato l'istituto dell'iniziazione, il soggiorno nel "castello solitario" diviene pauroso, e l'originario dio infero diviene il temibile sposo che non si vede o non si deve vedere. È una forte alterazione negativa; ma qualcosa dell'antico valore positivo di quell'esperienza rimane: lo sposo mostruoso o bestiale si rivela poi spesso, nelle favole, molto meno brutto e pericoloso di quanto si temesse» (Jesi 2010: 88-89).
} 
cosciente, rappresenta nonostante tutto una forma di ingenuità, che limita la presunta "superiorità" del parodista e consente l'affiorare del mito» (Jesi 2002: 195). E citando ancora da Mann, Jesi scrive che la parodia è «l'orgoglioso sgambetto alla sterilità con cui lo scetticismo, $[\ldots]$ la sensibilità per il mortifero diffondersi della banalità, minacciano i grandi ingegni» (Jesi 2002: 190).

La definizione di parodia come «orgoglioso sgambetto» ci consente di inserire nella serie terminologica morantiana oggetto della nostra interpretazione anche la parola "scandalo", che, com'è noto, nella sua etimologia greca, indica propriamente l'insidia, l'ostacolo che fa inciampare e cadere. Come Elsa Morante scrive nel saggio Pro o contro la bomba atomica, la presenza stessa dello scrittore è sempre uno «scandalo» (Morante 2001c: 1549). Sarebbe una operazione poco utile indicare tutte le occorrenze del termine scandalo negli scritti di Elsa Morante, perché è ormai noto che si tratta di una vera e propria parola-chiave della sua poetica. Appare assai significativo che fra queste occorrenze ve ne sia una riferita al padre di Arturo, che, come abbiamo già visto, è l'incarnazione del mistero, nella sua doppiezza demonica, e l'allegoria della parodia. È lo stesso Wilhelm a dire di sé: «IO SONO UNO SCANDALO» (Morante 2001a: 1101).

Lo scandalo del padre di Arturo, il suo mistero, la sua ambiguità rivelano la doppiezza e l'irresolutezza di un luogo fuori dall'universo mitico, di cui tuttavia si conserva la nostalgia. La parodia, come canto parallelo, ridesta l'eco del mistero che ciascuno porta dentro di sé in quanto essere vivente ormai fuori dal limbo. Era stato sempre l'Amalfitano a profetizzare che il padre di Arturo, nato da genitori di diversa nazionalità, portava «due sangui diversi nelle vene» e quelli che vivono questa condizione «non trovano mai riposo né contentezza». Egli aveva detto a Wilhelm: «Tu te ne andrai da un luogo all'altro, come se fuggissi di prigione, o corressi in cerca di qualcuno; ma in realtà inseguirai soltanto le sorti diverse che si mischiano nel tuo sangue, perché il tuo sangue è come un animale doppio, è come un cavallo grifone, come una sirena» (Morante 2001a: 1016).

In Menzogna e sortilegio, Anna Massia è esplicitamente definita «dèmone» (Morante 2001b: 650) e anche questo personaggio è descritto, per un verso, come l'oggetto di venerazione divina da parte della figlia Elisa, che la definisce la «Beata» (Morante 2001b: 184), per l'altro come la perenne preda di una insana e ossessiva passione che la porta a consumarsi d'amore per uno spettro. Anche qui, come nell'Isola di Arturo, il mistero di Anna deriva soprattutto dalla sua doppia nascita, per metà umile e per metà aristocratica. Anna, «gelosa guardiana dei propri misteri» (Morante 2001b: 849), è preda di una «nervosa malinconia» determinata dall'amore infelice per il nobile cugino Edoardo, «divinità vanesia e capricciosa» (Morante 2001b: 797), e «labile despota» (Morante 2001b: 862). Il delirio di Anna è descritto nel romanzo come l'effetto del vapore lunare di «astri melanconici»e, scrive l'autrice, «quei vapori lunari ed erratici sono i soli numi della mia epopea familiare, e Anna, la più bella, rimase fedele ad essi fino alla morte» (Morante 2001b: 921-922). Ma anche i «tristi capricci» (Morante 2001b: 604) di Edoardo sono il segno del suo essere «un personaggio quasi divino» (Morante 2001b: 765), un «puledrino d'una razza privilegiata» (Morante 2001b: 767). 
In esseri della specie di Edoardo, che rifuggono dal riposo come da un simbolo di morte, noi possiamo, credo, riconoscere dei cittadini del Paradiso Terrestre non ancora acclimatati all'esilio. La vita mortale, rinchiusa fra il tempo e lo spazio, è una prigione per loro, che incessantemente si agitano fra queste angustie illusi forse di ripercorrere $\mathrm{i}$ liberi campi originari. Si direbbe che del frutto gustato da Adamo toccò ad essi in eredità solo il sapore della condanna, non quello della conoscenza. La libertà, difatti, cui essi aspirano, è soltanto quella dell'Eden, gli altri, e più veri, paradisi sono ad essi negati. E la loro agitazione effimera ci richiama alla mente certe inquiete, patetiche belve, che, tolte dalla foresta nativa e poste in gabbia, senza tregua van correndo da una rete di sbarre all'altra. Ché la libertà perduta le incalza, e la loro sorte di fiere nega ad esse il santo riscatto d'Adamo (Morante 2001b: 214).

Dall'amore per un universo perduto e non più raggiungibile nasce la parodia come scandalo, sgambetto alla triste mescolanza della condizione umana, alla intrinseca contraddizione di esseri perennemente desideranti e mancanti. L'epilogo di Menzogna e sortilegio, che comprende un commiato in versi per il Gatto Alvaro, il cui «mistero» (Morante 2001b: 17) si annuncia appunto solo alla fine, ricompone in una costellazione la irrequieta quête d'amore del romanzo. A proposito di Anna, Edoardo e Francesco si legge:

M'immagino che là dove essi dimorano, e dove certo tutt'altre leggi da quelle della terra regolano amori e nozze, essi avranno potuto formare tutti e tre insieme una famiglia, godendo senza peccato del loro reciproco, triplice amore. Acceso da questo amore fraterno, forse il loro innocente trio familiare forma a quest'ora nel firmamento una costellazione, che sarà senza dubbio detta del Cugino (Morante 2001b: 942).

Del resto il genere romanzo, sempre secondo l'interpretazione di Carchia, si caratterizza come «evocazione magica della forma dal caos brulicante della vita» (Carchia 1983a: 16), alla cui descrizione esso si rivolge. Perciò, «in quanto fuga della forma da se stessa», esso ruota intorno «alla potenza primigenia che incessantemente rinnova la vita: la potenza dell'amore»; e «l'amore è l'anelito stesso di questa forma - aperta e disgregata - verso un compimento irraggiungibile» (Carchia 1983a: 17). Che gli amori infelici e impossibili siano la materia di tutti i romanzi di Elsa Morante è un dato che deve essere letto senz'altro al di là della semplice questione tematica, dal momento che evidentemente l'impossibilità del raggiungimento dell'oggetto amato, la quête frustrata, è in Elsa Morante ricca di significati più profondi che investono la natura stessa dell'oggetto ricercato. In Menzogna e sortilegio ella scrive che «non è umiliante amare chi non ci ama, né servire chi è grande, perfetto, e vale più di noi; ma scoprire che l'oggetto da noi rispettato e venerato, e servito come il nostro Dio, come il segno ultimo cui tende il nostro orgoglio; scoprire che questo oggetto era una cosa spregevole, ecco la massima umiliazione che possa toccarci» (Carchia 1983a: 841).

In Menzogna e sortilegio l'amore folle per Edoardo, che coinvolge contemporaneamente Anna, Elisa e Concetta, la madre di Edoardo, impazzita per la 
morte del figlio, è alimentato da un falso epistolario, cioè da una serie di lettere scritte da Anna stessa e attribuite al defunto Edoardo. Ė Elisa ad ammettere che il finto epistolario si trasforma per lei «in uno specchio, in cui l'amato viso di Anna [...] appare così imbruttito e stravolto, che memoria, volontà e fantasia [...] suggeriscono di scegliere una menzogna in luogo di [una] diagnosi» (Morante 2001b: 787).

Ma il romanzo in cui la vacuità della quête d'amore emerge con più desolazione è senz'altro 1'ultimo: Aracoeli. La ricerca del mistero divino della maternità, evocato dal nome che dà il titolo al romanzo, è una tragica parodia del viaggio di Ulisse. Il figlio di Aracoeli, Manuele, ritiene di essere «un finto Ulisse di terra, viaggiante fra finti vivi incantati da finte musiche verso colonne d'Ercole anch'esse finte» (Morante 2001d: 1201). La ricerca della madre, dopo la sua morte, nel suo luogo natale in Andalusia, è un viaggio verso un luogo che si chiama El Almendral, il cui nome evoca un mandorleto; qui, tuttavia, Manuele troverà soltanto «una sassaia bruciata dal vento» (Morante 2001d: 1192-1193). Quella del figlio è «una domanda d'amore ormai scaduta e inservibile, ma ostinata fino all'indecenza» (Morante 2001d: 1171), perché «la più nera infelicità terrestre» è quella di «esistere vivi dove non c'è nessuno che ci ama» (Morante 2001d: 1398). Per lui la perdita dell'amore materno ha significato la cacciata dall'Eden a cui non riesce a rassegnarsi. Si legge in Aracoeli:

Eravamo integri, prima della Genesi; e può darsi che la cacciata dall'Eden vada intesa, nel suo senso occulto, per un gioco ambiguo e provocatorio: «Avete mangiato il frutto proibito», dice la sentenza del Signore, «ma non quello segreto della vita, che io, Padrone del giardino, vi tengo nascosto, perché vi renderebbe uguale agli dèi» (Morante 2001d: 1289).

L' «integrità deificante» è data dal fatto che «gli dèi non sono maciullati dalla macchina dei sensi. Sono integri. Passato e presente e futuro - tenebre e luce morte e vita - i multipli e gli addendi - i diversi e i contrari - per loro sono tutti uno» (Morante 2001d: 1289). La nascita è di per sé la rottura di quella che Manuele definisce «congiunzione inseparabile» (Morante 2001d: 1189) e l'esistenza è una «macchia informe» (Morante 2001d: 1213) il cui percorso è simile a quello che avviene su una scala in cui si trova ad ogni tratto «un sasso che $[. .$.$] fa inciampare»$ (Morante 2001d: 1248). La ricerca del mistero della sua "mater purissima" è anche lo scandalo della scoperta della ninfomania di Aracoeli, ribaltamento parodico del mistero mariano. Manuele ammette che «il [suo] tesoro era un mistero» e che «l'intelligenza contamina i misteri». Pertanto, la preghiera che rivolge alla madre morta recita così:

È stata la tua misteriosa ambiguità, Aracoeli, che mi ti ha resa immortale; [...] e così ti ringrazio per il nostro intrigo puerile. La tua terribile ambiguità [...], tuo scandalo tuo splendore, mi accompagnerà, giocando, al traguardo del vuoto. Che tu sia benedetta, mamita, per il tuo alibi (Morante 2001d: 1404). 
Il morbo che devasta il corpo di Aracoeli, che muore nel 1939, e di cui non rimarrà nemmeno la spoglia, a seguito del bombardamento del cimitero del Verano a Roma in cui ella era seppellita, è una evidente metafora della strage del secondo conflitto bellico. Abbiamo finora tenuto in ombra quanto il nostro discorso sulla parodia come cifra interpretativa della scrittura morantiana abbia a che fare con la riflessione sulla storia. Che essa, soprattutto la Seconda Guerra Mondiale, sia fondamentale per comprendere i quattro romanzi morantiani, e non solo La Storia in cui l'argomento viene posto al centro, è una questione che può essere compresa se riflettiamo più a fondo sulla nozione di parodia che abbiamo tentato di ricostruire in questa sede. Nell'Isola di Arturo la storia irrompe nella vicenda alla fine del romanzo, quando comprendiamo che l'abbandono dell'isola da parte di Arturo è la partenza per la guerra. Non immediatamente evidente sembra quale sia il riferimento ad essa in Menzogna e sortilegio, ma forse il romanzo può essere interpretato diversamente se si presta maggiore attenzione, fra le altre possibili considerazioni, e al di là della sua vicenda filologica, all'importanza di una dichiarazione fatta dalla Morante nel 1968, secondo cui l'idea di Menzogna e sortilegio sarebbe nata da quanto aveva sentito dire intorno «a una vecchia signora cieca a cui avevano ucciso il figlio in guerra [...] e a cui i famigliari nascondevano la verità», leggendole delle lettere e facendole credere che fossero del figlio (Morante 2001e: LVII ${ }^{6}$. Il romanzo, pertanto, trasfigurerebbe in una passione fatale e demonica quella condizione di perenne angoscia avvertita da una umanità consegnata alla storia e alla sua cieca violenza, il cui esito più disastroso è la guerra.

Se attingiamo ancora una volta alla nozione di parodia come cifra interpretativa del genere romanzo, possiamo ricavare da Carchia l'idea che il dominio in esso del misterico, con quello che ciò implica, ovvero che «l'umano possa divenire il veicolo del divino, che la terrestrità ospiti l'inafferrabile natura celeste», rimanda al fatto che «il campo della storia si apre proprio con la trasfigurazione del presente allorché la [sua ] temporalità [...], del tutto irrilevante a fronte del [...] "passato assoluto" del mito, tende a configurarsi come lo spazio evenemenziale del divino». In altri termini, il mistero è la parodia del mito se si considera che in esso si manifesta l'origine della storia intesa come particolare spazio di azione dell'umano, derivante dall'incapacità di vivere in un mondo senza dèi. Nell'idea di storia dell'uomo che nasce con il mistero, dunque, la «sostanzialità mitica» può tornare a manifestarsi soltanto parodicamente all'interno di una «quotidianità svuotata di senso» (Carchia 1983a: 25).

Il romanzo La Storia, «uno scandalo che dura da diecimila anni», deve il suo avvio proprio a quello che la protagonista Ida definisce lo «scandalo supremo impensato» (Morante 2001f: 354), ovvero lo stupro di un soldato tedesco che la lascia incinta di un bimbo i cui occhi, dello stesso colore di quelli del padre, sono «il turchino del suo scandalo» (Morante 2001f: 366). La storia del romanzo, cioè,

${ }^{5}$ Si tratta di una intervista rilasciata a Michel David e apparsa su «Le Monde» il 13 aprile 1968 . 
nasce dalla parodia del mistero della natività, che ad Ida viene annunciata in un sogno ambientato in una «foresta di piantagioni favolose» (Morante 2001f: 353). Il piccolo Useppe è, però, troppo bello e fragile per stare al mondo: la sua è una «diversità favolosa» (Morante 2001f: 833) destinata ad essere travolta dal Grande Male, sviluppatosi dopo aver visto le foto dei lager e dei partigiani impiccati. Ma la parodia del mito è anche qui l'inciampo che rivela l'amore per l'oggetto parodiato, alla cui integrità si guarda con nostalgia. Dopo ciascun attacco epilettico, sul volto del bambino è destinato a tornare un «sorriso d'un ciecolino» (Morante 2001f: 880), «un sorrisetto incantato di guarigione» (Morante 2001f: 800); e un sorriso è anche quello che Useppe vede in sogno sul volto di sua madre. Vi era solo il volto di Ida con gli occhi chiusi; d'un tratto si sentiva un urlo, ma «la faccia di Ida, sempre con gli occhi chiusi, e con la bocca pure chiusa, intanto s'era messa a sorridere» (Morante 2001f: 719). Jesi, tenendo conto degli studi di Kerényi sul «riso degli dèi», riflette sul fatto che esso è «il simbolo più alto e misterioso dell'abisso che separa la natura divina da quella umana», ovvero «l'emblema di un'esistenza la cui serenità olimpica è inaccessibile all'uomo» (Jesi 2002: 198).

Ci sembra, infine, che l'estetizzazione del quotidiano, la sua trasfigurazione mitica, messa in atto per il tramite della parodia, interroghi proprio la contraddizione insolubile di un'esistenza storica condannata alla violenza insensata della guerra, definita, nel romanzo, «un'algebra sconclusionata» (Morante 2001f: 274), dato che la Seconda Guerra Mondiale rappresenta «la peggiore oscenità di tutta la Storia». Essa, dice Davide Segre nel romanzo, «è tutta un'oscenità fino dal principio, però anni osceni come questi non ce n'erano mai stati» (Morante 2001f: 943).

In conclusione, l'originalità della nozione di parodia nei romanzi della scrittrice ci sembra consistere nel particolare rapporto che essa intrattiene da una parte con il mito e dall'altra con la storia, intesa come successione violenta di ingiustizia e inumanità che trova il suo esito più tragico nella guerra mondiale.

\section{RIFERIMENTI BIBLIOGRAFICI}

Agamben, Giorgio (2005): Parodia, in Profanazioni, Roma, Nottetempo.

AgAmBEn, Giorgio (2010): La ragazza indicibile. Mito e mistero di Kore, Milano, Electa.

CARCHIA, Gianni (1983a): «La nascita del romanzo», in Rivista di estetica, 13, pp. 15-31; e in Carchia, Gianni (1983b): Dall'Apparenza al Mistero. La nascita del romanzo, Milano, Celuc.

D’ANGeli, Concetta (2003): Leggere Elsa Morante. "Aracoeli", "La Storia”, "Il mondo salvato dai ragazzini", Roma, Carocci.

Dell'AiA, Lucia (2013): La sfera del puer. Il tempo dei ragazzini di Elsa Morante, Roma, Aracne.

Dell'AiA, Lucia (2012a): La Storia e l'affabulazione, in Sgavicchia, Siriana (ed.): La Storia di Elsa Morante, Pisa, Edizioni ETS, pp. 183-189. 
Dell'Aia, Lucia (2012b): Parodia e Profanazione, in Studi su Agamben, Milano, Ledizioni, pp. 24-32.

GENETTE, Gerard (1997): Palinsesti, Torino, Einaudi.

JESI, Furio (2010): «Trasmissione sulla favolistica», in Riga, 31.

JESI, Furio (2002): Parodia e mito nella poesia di Ezra Pound, in Letteratura e mito, Torino, Einaudi.

MoRANTE, Elsa (1988 [2001a]: L'isola di Arturo, in Opere, Milano, Mondadori, vol. I.

MORANTE, Elsa(1990[2001b]: Menzogna e sortilegio, in Opere, Milano, Mondadori, vol. I.

MoRANTE, Elsa (2001c): Pro o contro la bomba atomica, in Opere, Milano, Mondadori, vol. II.

MorAnTE, Elsa (2001d): Aracoeli, in Opere, Milano, Mondadori, vol. II.

MORANTE, Elsa (2001e): Cronologia, in Opere, Milano, Mondadori, vol. II.

MorAnTE, Elsa (2001f): La Storia, in Opere, Milano, Mondadori, vol. II. 